\title{
PITUITARY INCIDENTALOMAS - WHERE DO WE GO FROM HERE?
}

"Begin at the beginning," the King said, very gravely, 'and go on till you come to the end: Then stop.' - Lewis Carroll, Alice in Wonderland

Pituitary incidentalomas (PIs) are generally described as sellar lesions without any overt features of pituitary disease that is found on brain imaging done for unrelated conditions. ${ }^{[1]}$ After their initial description in $1936,{ }^{[2]}$ various studies have reported the prevalence of PI to be around $3-27 \%$ in autopsy series ${ }^{[3-5]}$ and around $10 \%$ on diagnostic imaging. ${ }^{[6]}$

However, despite their prevalence, limited information is available on the natural history of PI and many key questions remain unanswered. This article will briefly explore some of those questions and the associated inconsistencies.

The data from the autopsy studies showed that vast majority of PI were microadenomas $(<10 \mathrm{~mm})$, whereas those found on imaging typically tend to be macroadenomas $(>10 \mathrm{~mm})$. Our own study, ${ }^{[7]}$ which was based on radiological imaging, showed that $71 \%$ of PI presented as macroadenomas, a trend supported by other studies. ${ }^{\left[{ }^{[8,9]}\right.}$ This discrepancy is likely due to the imaging sequence as most PI are identified on imaging (magnetic resonance imaging or computed tomography) of the brain as opposed to the dedicated imaging of the sella.

Although PIs, by definition, are found on brain imaging done for unrelated conditions, the question remains if they are truly incidental lesions. Our own series ${ }^{[7]}$ and another one from Japan ${ }^{[8]}$ reported fairly similar indications for primary brain imaging, which included headache, dizziness, TIA/stroke, seizures and trauma. Headache has been associated with both small and large pituitary tumours $^{[9]}$ and can also occur in functioning pituitary tumours such as growth hormone-producing adenomas.

Furthermore, some PI on presentation may turn out to have previously undiagnosed visual field abnormalities. ${ }^{[7]}$ In a

Correspondence: Syed Ali Imran, Department of Medicine,

Division of Endocrinology and Metabolism,

Dalhousie University, Halifax, Canada.

Email:simran@dal.ca recent analysis, ${ }^{[10]}$ we found that PIs were around 20\% more likely to present with previously undiagnosed visual field abnormalities than those who presented with clinical evidence of pituitary dysfunction. Can dizziness, which was a common indication for imaging, be associated with partial loss of vision in this group remains a tantalizing research question. As these associations become clearer, it would not be unreasonable to revisit the current definition of PI.

Although most PI tend to be either clinically nonfunctioning pituitary adenomas or non-pituitary sellar masses, around $22 \%$ of all patients in our series ${ }^{[7]}$ had previously undiagnosed functioning adenomas include prolactinomas $(15 \%)$, growth hormone-producing adenomas (5\%) and ACTH-producing adenoma (3\%). Furthermore, most of these $(>75 \%)$ were already macroadenomas at presentation. This is a surprising trend, as these tumours despite their size tend to be relatively hormonally quiescent. It remains to be determined why these tumours behave differently from their clinically manifesting counterparts. Data from other studies are rather conflicting; for instance, a large study from Japan following 506 patients ${ }^{[8]}$ reported no clinical or biochemical evidence of hyperfunctioning tumours, whereas another series from Greece ${ }^{[11]}$ reported $21 \%$ of patients had functioning tumours. Further studies looking at the pathophysiological characteristics of functioning PI versus clinically manifesting pituitary adenomas are needed. It is well described that most non-aggressive and slow-growing pituitary adenomas tend to be densely granulated adenomas as opposed to the sparsely granulated adenomas that are clinically and biochemically more aggressive. ${ }^{[12]}$ It is currently unclear if functioning PIs are more likely to be densely granulated, and thus, as a result presents without overt clinical features of hormonal dysfunction. Similarly, studies on molecular genetics of these two entities are also required to identify key transcription factors that may explain their differential behaviour.

The risk of previously undiagnosed hormonal dysfunction is considerable in patients with PI. Indeed, our own data $^{[7]}$ showed that almost $30 \%$ of patients with PI had 
biochemical evidence of hormonal loss at presentation including growth hormone deficiency, hypogonadism, secondary hypothyroidism, secondary hypoadrenalism and even diabetes insipidus. Although no study has formally compared the need for surgery in this group, our own analysis ${ }^{[10]}$ showed that almost a third (32\%) of PI eventually required surgical management as opposed to $46 \%$ of the clinically manifesting pituitary adenomas. Whether long-term outcomes of PI with or without treatment are different, remain to be studied. Future studies need to assess the rate of remission of incidental versus clinically manifesting pituitary tumours in response to medical and surgical therapy.

It is well established that larger and more aggressive functioning pituitary adenomas are less likely to achieve remission and where most PI present as macroadenomas, is the rate of remission similarly low? In addition, the pattern of response of functioning PI to the currently available pharmacologic therapies is another key area that needs to be explored. These are important considerations given that expert pituitary surgeons may not be available in certain areas; therefore, it is crucial to identify the most optimal treatment strategy for these growths.

Another area of uncertainty is the long-term rate of morbidity and mortality in functioning PI. The risk of morbidity and mortality is significantly high in clinically manifesting functioning tumours associated with acromegaly and Cushing's disease. In addition, studies have reported a high risk of lingering psychological sequelae associated with these disorders even after achieving biochemical remission. ${ }^{[13,14]}$ Given the relatively indolent presentation of these functioning PI, it is important to determine the risk of associated long-term complications, particularly risk of malignancy, sleep apnoea, hypertension and sexual dysfunction. These risks will help determine the targets for therapeutic intervention.

A familial predisposition of certain pituitary adenomas is being increasingly recognised. Is the risk of PI commoner in patients with known germline or somatic mutations are also an area that needs to be explored. One of the common mutations is a germline heterozygous mutation in the aryl hydrocarbon receptor-interacting protein ${ }^{[15]}$ that is typically associated with large, invasive pituitary (mostly somatotropic or somatolactotropic) adenomas.
Therefore, it is suggested that younger patients presenting with larger pituitary adenomas should undergo genetic testing. It would be crucial to identify such mutations in patients presenting with PI, which would eventually allow physicians to refine strategies for earlier testing.

In summary, PIs are frequently encountered in clinical practice and with a more widespread utility of sensitive imaging techniques; it is likely that the prevalence will continue to rise. Therefore, it is crucial to learn about the natural history of these lesions, to develop appropriate management paradigms. Furthermore, detailed histopathological, genetic and molecular studies are needed to understand the differences in characteristics at presentation and response to management therapies.

Syed Ali Imran

Department of Medicine, Division of Endocrinology and Metabolism, Dalhousie University, Halifax, Canada Received: 2 July 2016 / Accepted: 22 August 2016

\section{References}

1. Freda PU, Beckers AM, Katznelson L, et al. Pituitary incidentaloma: An endocrine society clinical practice guideline. J Clin Endocrinol Metab 2011;96:894-904.

2. Costello RT. Subclinical adenoma of the pituitary gland. Am J Pathol 1936;12:205-16.1.

3. Chacko AG, Chandy MJ. Incidental pituitary macroadenomas. Br J Neurosurg 1992;6:233-6.

4. Reincke M, Allolio B, Saeger W, et al. The 'incidentaloma' of the pituitary gland. Is neurosurgery required? JAMA 1990;263:2772-6.

5. Teramoto A, Hirakawa K, Sanno N, et al. Incidental pituitary lesions in 1,000 unselected autopsy specimens. Radiology 1994;193:161-4.

6. Hall WA, Luciano MG, Doppman JL, et al. Pituitary magnetic resonance imaging in normal human volunteers: Occult adenomas in the general population. Ann Int Med 1994;120:817-20.

7. Imran SA, Yip CE, Papneja N, et al. Analysis and natural history of pituitary incidentalomas. Eur J Endocrinol 2016;175:1-9.

8. Sanno N, Oyama K, Tahara S, et al. A survey of pituitary incidentaloma in Japan. Eur J Endocrinol 2003;149:123-7.

9. Levy MJ, Matharu MS, Meeran K, et al. The clinical characteristics of headache in patients with pituitary tumours. Brain 2005;128:1921-30.

10. Vaninetti N, Munro V, Clarke DB, et al. Clinically Manifesting vs. Incidental Sellar Masses-A Comprehensive Comparative Study. Ottawa, Ontario: $19^{\text {th }}$ Annual CDA/ CSEM Professional Conference; 2016. 
11. Anagnostis P, Adamidou F, Polyzos SA, et al. Pituitary incidentalomas: A single-centre experience. Int J Clin Pract 2011;65:172-7.

12. DeLellis RA, Lloyd RV, Heitz PU, et al. Pathology and genetics of tumors of endocrine organs, World Health Organization Classification of Tumors. Lyon: IARC Press; 2004. p. 9-45.

13. Imran SA, Tiemensma J, Kaiser SM, et al. Morphometric changes correlate with poor psychological outcomes in patients with acromegaly. Eur J Endocrinol 2016;174:41-50.

14. Tiemensma J, Daskalakis NP, van der Veen EM, et al. Drawings reflect a new dimension of the psychological impact of long-term remission of Cushing's syndrome. J Clinic Endocrinol Metabol 2012;97:3123-31.

15. Korbonits M, Storr H, Kumar AV. Familial pituitary adenomas-who should be tested for AIP mutations? Clinic Endocrinol 2012;77:351-6. 